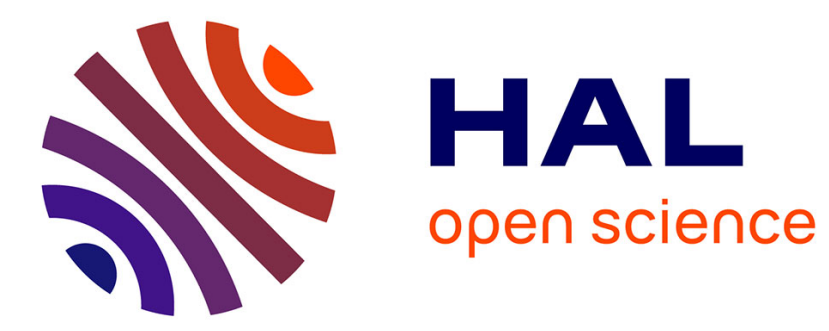

\title{
Parameterizable constant temperature anemometer: a new method for the analysis of velocity temperature coupling in turbulent heat transfer
}

\author{
M. Ndoye, Joël Delville, Dominique Heitz, G. Arroyo
}

\section{- To cite this version:}

M. Ndoye, Joël Delville, Dominique Heitz, G. Arroyo. Parameterizable constant temperature anemometer: a new method for the analysis of velocity temperature coupling in turbulent heat transfer. Measurement Science and Technology, 2010, 21 (7), p. - p. 10.1088/0957-0233/21/7/075401 . hal-00515606

\author{
HAL Id: hal-00515606 \\ https://hal.science/hal-00515606
}

Submitted on 7 Sep 2010

HAL is a multi-disciplinary open access archive for the deposit and dissemination of scientific research documents, whether they are published or not. The documents may come from teaching and research institutions in France or abroad, or from public or private research centers.
L'archive ouverte pluridisciplinaire HAL, est destinée au dépôt et à la diffusion de documents scientifiques de niveau recherche, publiés ou non, émanant des établissements d'enseignement et de recherche français ou étrangers, des laboratoires publics ou privés. 


\title{
Parameterizable Constant Temperature
}

\section{Anemometer, a new method for the analysis of velocity-temperature coupling in turbulent heat transfer}

\author{
M. Ndoye ${ }^{1,2,3}$, J. Delville ${ }^{1}$, D. Heitz ${ }^{2,3}$ and G. Arroyo ${ }^{2,3}$ \\ ${ }^{1}$ Institut PPRIME, Département Fluide Thermique et Combustion, \\ CNRS - Université de Poitiers - ENSMA UPR 3346, F-86036 Poitiers Cedex, France \\ ${ }^{2}$ Cemagref, UR TERE, F-35044 Rennes, France \\ ${ }^{3}$ Université européenne de Bretagne, France \\ E-mail: malick.ndoye@lea.univ-poitiers.fr
}

\begin{abstract}
A novel anemometer was designed and implemented for simultaneous measurement of velocity and temperature in air flows with a single hot wire probe. The principle of periodically varying the overheat ratio of the wire was selected and applied through a parameterizable electronic chain. The specific methods developed for the calibration procedure and the signal processing are explained. The accuracy of the measurements was assessed by means of Monte Carlo simulations. Examples of results are given for a non-isothermal low speed mixing layer. Finally, the ability to extract velocity-temperature coupling from a turbulent non-isothermal flow is highlighted through a conditional Probability Density Function (PDF) analysis.
\end{abstract}

Keywords: Hot wire anemometry, velocity measurements, temperature measurements, synchronic measurements, heated turbulent flow, Monte Carlo method

Submitted to: Meas. Sci. Technol. 


\section{Introduction}

The simultaneous measurement of temperature and velocity in unsteady turbulent flow configurations at a given location remains an unresolved problem, particularly if a good frequency response range is required. Apart from its fundamental applications, the solution to this problem is important in many industrial applications. Among the methods reported in the literature for unsteady flows, the Constant Temperature Anemometer (CTA) provides a possible approach to carrying out accurate measurements of velocity (with high overheat ratios) and temperature (with low overheat ratios) (Bruun [6], Perry [16]). Different runs, corresponding to different selected overheat ratios, provide access to statistical quantities for both variables, i.e. mean, RMS, correlations and even spectra or cross-spectra. This method has been used to provide relevant comparisons with data originating from numerical simulations based on Reynolds Averaged Navier Stokes methods (RANS). However, the latter approach cannot be used as far as instantaneous velocity-temperature distributions are concerned: for example joint velocity-temperature distributions cannot be obtained in this way. This point is crucial when unsteady situations have to be addressed, with a need to analyze the unstationary behavior of the flow through instantaneous velocity-temperature distributions and cross-correlations (coupling unsteady simulations and experimental data, control, etc.). In this case, the most common approach in hot wire anemometry is either the use of one or more hot wire probes operating in constant temperature modes, combined with a cold wire operating in constant current mode, or two hot wires with two different overheat ratios placed close to each other. However, this method, used among others by Blair and Bennett [3], Lienhard and Helland [8], Vukoslavcevic and Wallace [18], Bremhorst and Graham [4], lacks spatial resolution, and interference from the wires is not easy to overcome.

A special thermo-anemometer has been designed to address this problem, the main characteristic of this novel approach being to make the overheat ratio of the wire continuously change in a way that constantly repeats a given pattern with a frequency $f$. If the period $\tau=1 / f$ is small enough compared to the time scale of the phenomenon observed, temperature and velocity can be considered as obtained at the same time from the same single sensor, giving access for example to joint velocity-temperature probability distributions and velocity-temperature correlation. This thermo-anemometer, in which the overheat ratio can be changed in real time, is hereafter called a PCTA (Parameterizable Constant Temperature Anemometer). The method and device are based on the constant hot wire anemometry technique and apply the multiple overheat principle.

We also propose an original non-stationary velocity-temperature calibration approach. This method responds to: i) the need to calibrate the hot wire probes for velocity and temperature for use in a flow in the presence of spatio-temporal variations of the temperature due to turbulence, and $i i$ ) the need to calibrate the hot wires rapidly 
to avoid any variation in flow characteristics as encountered in classical calibration methods, which are relatively long and generally need to stabilize the flow in terms of velocity and temperature. Our approach allows calibration in a wide velocity-temperature range in a single run of a few minutes.

In this paper, we first present the PCTA principle and the specific electronic circuit implemented to produce variations in wire overheat in very short periods, then the calibration procedure is described, after which we set out the specific algorithms developed to process the PCTA signal and quantify the uncertainty by Monte Carlo simulations. In the final part, some results of the validation of the PCTA in a non-isothermal mixing layer flow are detailed and discussed.

\section{Design and principle of the PCTA}

The PCTA thermo-anemometer presented here consists of a $2.5 \mu \mathrm{m}$ diameter and $1.2 \mathrm{~mm}$ long Pt-W wire connected to an anemometer. A simplified circuit diagram is shown in figure 1. This anemometer card was developed by Laboratoire d'Etudes Aérodynamiques

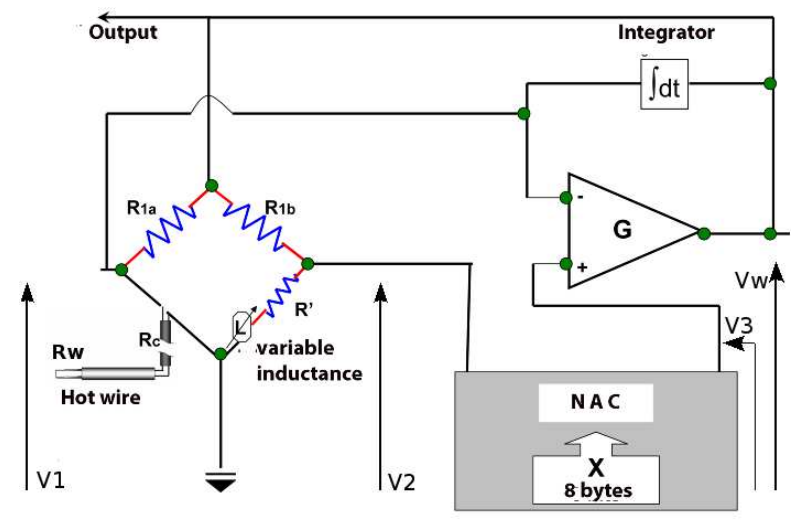

Figure 1. Simplified diagram of the PCTA anemometer card.

in Poitiers, France. In this device, to vary the overheat ratio of the wire $a_{w}$, defined as the ratio of the operating wire resistance $R_{w}$ to the wire resistance $R_{0}$ at ambient temperature, the output voltage of the resistance $R^{\prime}$ is controlled by an 8 bit digital-toanalog converter whose setting $X$ is scored between 0 and 255. At the bridge equilibrium (see figure 1), we have:

$$
\begin{aligned}
V_{1} & =\frac{V_{w}\left(a_{w} R_{0}+R_{c}\right)}{a_{w} R_{0}+R_{c}+R_{1 a}} \\
V_{2} & =\frac{V_{w} R^{\prime}}{R^{\prime}+R_{1 b}} \\
V_{3} & =\frac{V_{2} X}{256} \\
V_{w} & =\left(V_{3}-V_{1}\right) G .
\end{aligned}
$$


The byte value $X$ for the digital-to-analog converter is obtained by combining equations (1) to (4):

$$
X=\left(\frac{a_{w} R_{0}+R_{c}}{a_{w} R_{0}+R_{c}+R_{1 a}}+\frac{1}{G}\right)\left(\frac{R^{\prime}+R_{1 b}}{R^{\prime}}\right) 256 .
$$

This depends on the overheat ratio chosen and on the characteristics of the wire. For example, in a classical constant temperature anemometer, $R^{\prime}$ is a potentiometer able to satisfy the following condition:

$$
R^{\prime} \geq K\left(a_{w} R_{0}+R_{c}\right)
$$

where $K=\frac{R_{1 b}}{R_{1 a}}$ is the bridge ratio. For the PCTA anemometer, the voltage across $R^{\prime}$ is varied with the digital-to-analog converter. $R^{\prime}$, which is chosen for a maximum value of $a_{w}$ of 2 , must be adjustable up to values compatible with the probe characteristics $R_{0}$ and $R_{c}$. Typically, wires with a diameter greater than or equal to $2.5 \mu \mathrm{m}$ can be used for $R_{1 b}=120 \Omega, R_{1 a}=22 \Omega, R_{c} \simeq 2 \Omega$ and $R_{\max }^{\prime}=120 \Omega$.

The anemometer is entirely configurable through a user interface under computer control. The setting operations comprise the selection of a multiple overheat sequence pattern, measurement of the wire resistance $R_{0}$ at the current ambiant temperature, and the classical initial adjustment to the dynamic response of the wire. The wire can be submitted to sequences of two to eight overheats, each sequence constituting a pattern that can be repeated every 100 to $1000 \mu \mathrm{s}$, according to the wire diameter and flow velocity. Then a theoretical bandwidth of up to several $\mathrm{kHz}$ can be obtained.

A typical plot of the output voltage signal from the anemometer is illustrated in figure 2. We have chosen to decrease the wire overheat step by step for each sequence until the required minimum level is reached. The output voltage obtained between two overheat switches, corresponding to what we call an overheat stage, comprises a nonprocessable transitional period, corresponding to the instantaneous response of the wire to the overheat switch, and an active period during which the overheat is actually applied with the PCTA operating in constant temperature mode. Ideally, the voltage should look like a plateau during this active period, but we considered that good repeatability of a non-horizontal pattern is sufficient to extract accurate velocity and temperature values over each sequence. Inside the repeated sequence, the duration of the overheat stages can be chosen to be identical for all the overheat levels or to vary from one to another.

The number $N_{0}$ of overheat levels in each sequence remains constant during a calibration and measurement experiment. The total number of sequences in a run is denoted $N_{s}$. We allocate the subscript $i$ to the current sequence $\left(i=1, N_{s}\right)$ and the subscript $k$ to the overheat levels applied $\left(k=1, N_{0}\right)$. 
a)
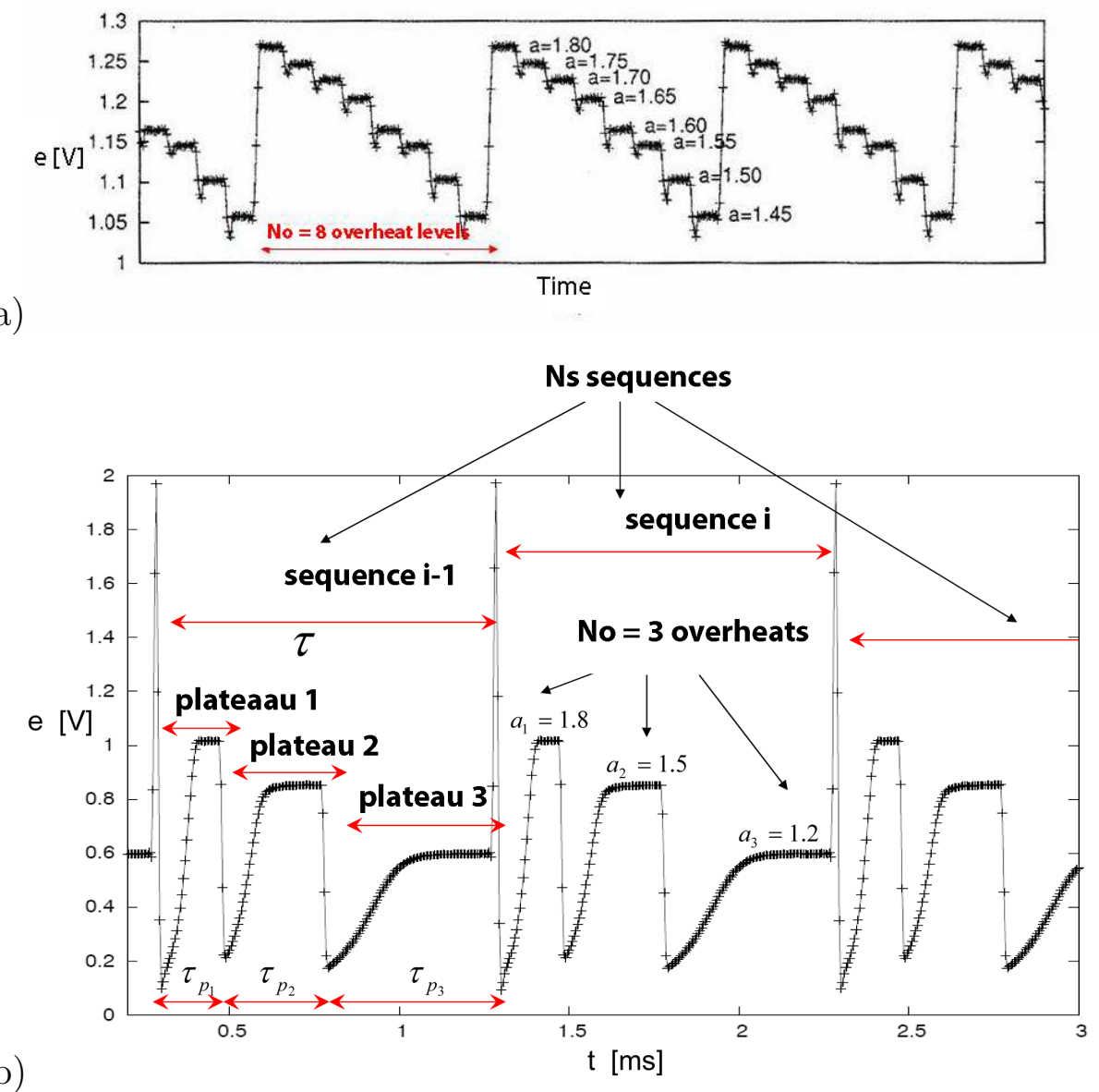

Figure 2. Output voltage obtained from the PCTA: a) typical time trace (obtained with $N_{o}=8$ overheat levels, b) notations and characteristic scales (example with $N_{o}=3$ overheat levels).

\section{Calibration}

The equations governing the response of a hot wire placed in a turbulent flow are generally derived from the energy equation of the wire. In cases of non-isothermal flow, various expressions have been used in the literature (e.g. Bruun [6] and Perry [16]). When the PCTA principle is used, the cooling equation of the wire, which relates the output voltage of the anemometer to the unknown velocity and temperature, is non-linear. In this study, we tested the following cooling equations:

- extended King's law (with temperature correction)

$$
e^{2}=\left(a+b U^{n}\right)\left(T_{w}-T\right)
$$

- polynomial law

$$
U=\sum_{i=0}^{4} \alpha_{i}\left(\frac{e}{\sqrt{T_{w}-T}}\right)^{i}
$$


where $e$ is the anemometric voltage measured, and $U$ and $T$ are the unknown instantaneous velocity and temperature of the flow. The other quantities, namely the wire temperature $T_{w}$, the coefficients $a, b$, (or $\left.\alpha_{i}\right)$ and the exponent $n$ need to be determined experimentally through a calibration process. It should be noted that, due to the multiple overheat principle, these coefficients are in fact vectors of which the components are their scalar values for each overheat level. Once these vectors have been determined through the calibration process, the velocity and temperature of the flow can be deduced for each individual multiple overheat sequence (subscripted $i=1, N_{s}$ ) from a combination of the output voltages measured $e_{i, k}$ (for $k=1, N_{o}$ ). The LevenbergMarquardt iterative method for resolution of non-linear equations was used to carry out the calibration.

\subsection{Strategy}

Because we wished to have a very short total calibration time (a few minutes), we chose to calibrate the probe in a non-stationary process by operating with continuously varying velocity and temperature conditions in order to determine the sensitivity of the measurement system to the velocity and temperature in a single run. To do so, we permanently varied the velocity and temperature of the flow at a pace compatible with the time constants of the reference probes. To separate the influences of temperature variations and velocity fluctuations, the flow velocity was varied in a sawtooth mode, while the flow temperature was regularly decreased with a lower change rate by taking advantage of the thermal inertia of the closed circuit of the calibration. An example of the time variations applied to the velocity and temperature during calibration is given in figure 3, with three overheat levels in a low-speed flow. It can be seen in this example that the velocity variation was nearly linear for 13 velocity cycles, applied over 3 minutes, thus ensuring an equally probable velocity distribution. Furthermore, the decrease in temperature obtained was globally monotonic and covered a range of about $18 K$. However there were local plateaux related to velocity minima. Indeed, the thermal exchange coefficient of the heat exchanger of the calibration facility decreases considerably for very low velocities and the temperature thus tends to remain constant for a few seconds. This suggests a degree of weakness of our velocity-temperature calibration for very low velocities.

\subsection{Calibration procedure}

The calibration procedure is based on the correspondance between the voltage response of the device under calibration and the simultaneous measurements of the velocity and temperature of a flow by two reference probes, one a Pitot dynamic pressure probe and the other a fine thermocouple providing a measurement frequency of 100 Hertz. The hot wire and the reference probes are connected to the PCTA measurement chain for synchronization of the data acquisition .

For some types of flow with velocities greater than $5 \mathrm{~m} / \mathrm{s}$, the calibration can be 
a)

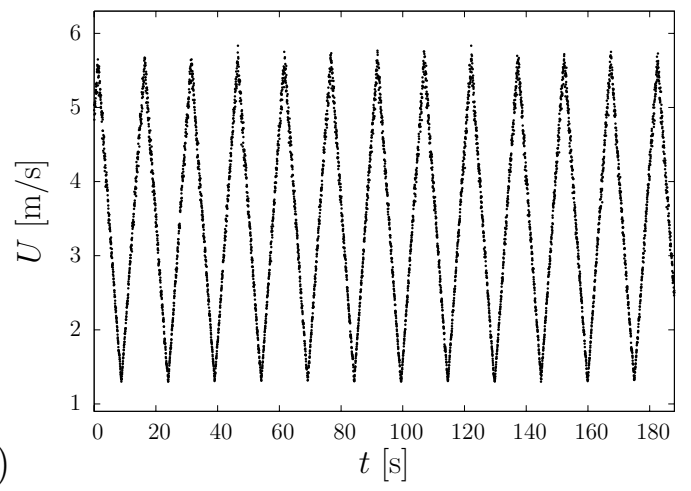

b)
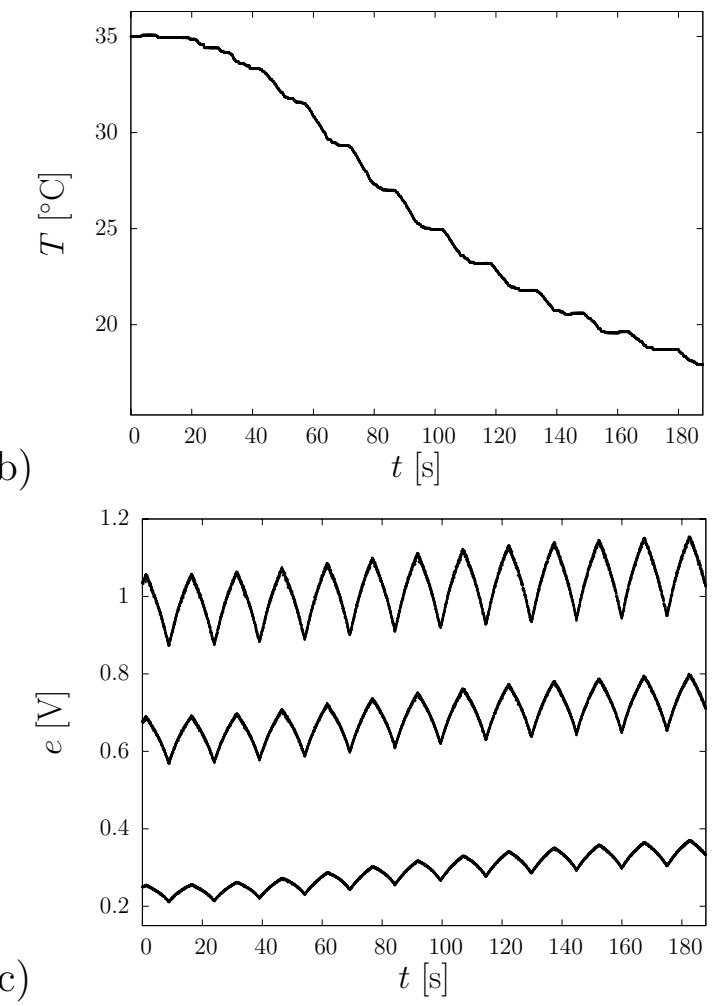

Figure 3. Example of temporal variations imposed on: (a) velocity, (b) temperature, (c) anemometer response during calibration $\left(N_{o}=3\right)$.

achieved in situ by placing the three probes in the potential part of the flow. An example can be found in our preliminary study [11] in a coaxial jet system where the hot wire being calibrated was placed in the potential core of the inner jet, which was initially heated above the ambient temperature.

Direct Pitot measurements were no longer suitable for the reference velocity measurement for lower velocities. Calibration was therefore performed in a small semiclosed loop wind tunnel (see figure 4 ) specially designed for this purpose. The test section (0.5 m long and $142 \mathrm{~mm} \times 142 \mathrm{~mm}$ in cross section) was supplied with a uniform inlet flow profile generated by a contraction cone with an adjustable velocity ranging from 0.5 $\mathrm{m} / \mathrm{s}$ to $5.5 \mathrm{~m} / \mathrm{s}$. The temperature change was controlled through an adjustable heater 


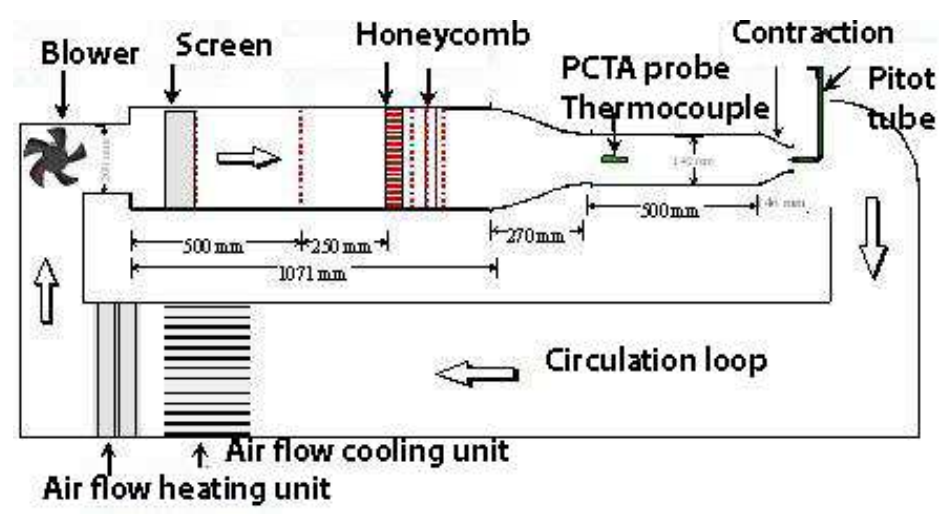

Figure 4. Diagram of calibration apparatus.

located upstream from the test section. A second profiled contraction cone was adapted at the downstream end of the test section to multiply the velocity by 9 at its outlet. The PCTA probe and the thermocouple were placed in the upstream part of the test section, while the Pitot tube was placed at the exit cross section of the downstream cone. The reference velocity in the test section was then calculated by applying the linear relation between the Pitot measurements and the actual velocity at the PCTA probe location. This relation was adjusted using PIV velocity measurements in the test section.

\section{Signal processing}

The algorithms developed to process the PCTA signals are presented in this section. They allow adjustment to a calibration law and simultaneous computation of velocity and temperature. Given that the calibration law used is non-linear, specific algorithms based on Levenberg Marquardt iterative methods [17] were developed for the following situations:

- Calibration: adjusting the calibration coefficients according to the data of a calibration set.

- Measurement: determination of the velocity and temperature of the flow from a group of anemometric voltage measurements with the PCTA, once the calibration coefficients have been calculated.

From here on we used only the extended King's law equation (which is bijective) to relate the output voltage to the unknown values of $T$ and $U$ in the flow. The polynomial law was used for measurements of $U$ and $T$ with very low velocities $(<1 \mathrm{~m} / \mathrm{s})$, appearing in this case to account more accurately for the thermal effects of the wire. The signal processing methods used were then similar to those explained below.

Inside each overheat sequence, the output voltage delivers a stepped pattern that represents step-by-step variations in the wire temperature $T_{w}$. This signal is first processed with filtering of the separately extracted active periods of the overheat 
plateaux to separate the overheats and provide a value of $e_{i, k}$ for each overheat level $k=1, \ldots, N_{0}$ of each overheat sequence $i$.

Classically an increase in overheat leads to an increase in the anemometric voltage and in sensitivity to velocity, while the sensitivity to temperature decreases, thus making it possible to separate the velocity and temperature measurements.

\subsection{Evaluation of calibration coefficients}

The procedure is here described for an extended King's law.

For each overheat level, the voltage measured is assumed to be related to the velocity and the temperature in the air by the equation:

$$
e_{i, k}^{2}=\left(a_{k}+b_{k} U_{i}^{n_{k}}\right)\left(T_{w, k}-T_{i}\right) .
$$

The second subscript $k$ is not assigned to $U_{i}$ and $T_{i}$, a basic hypothesis of the method being that the fluctuations in $U_{i}$ and $T_{i}$ inside each sequence can be ignored in order to extract a single pair $\left(U_{i}, T_{i}\right)$ through the processing of the $N_{o}$ voltage values $e_{i, k}$ in the $i^{t h}$ sequence. The consequence of this hypothesis is that velocity and temperature fluctuations of frequencies higher than $f=1 / \tau$ (see figure 2) will be filtered and the coefficients $a, b$, the exponent $n$ and the wire temperature $T_{w}$ are only subscripted $k$.

For each overheat level $k$, we seek the vector of parameters $\mathbf{A}_{\mathbf{k}}=\left(a_{k}, b_{k}, n_{k}, T_{w, k}\right)$ that best fits the calibration model predictions $f_{i, k}$ to the measured data $M_{i, k}$ by minimizing the squared difference between both quantities over the whole set of $N_{s}$ sequences through a Levenberg Marquardt process:

$$
\arg \min _{\mathbf{A}_{\mathbf{k}}} \quad\left\{\sum_{i=1}^{N_{s}}\left[M_{i, k}-f_{i, k}\left(U_{i}, T_{i}, \mathbf{A}_{\mathbf{k}}\right)\right]^{2}\right\},
$$

where

$$
f_{i, k}\left(U_{i}, T_{i}, \mathbf{A}_{\mathbf{k}}\right)=\left(a_{k}+b_{k} U_{i}^{n_{k}}\right)\left(T_{w, k}-T_{i}\right)
$$

is the model and $M_{i, k}=e_{i, k}^{2}$ is the PCTA squared output voltage measured for the selected overheat $k$. The minimization is achieved by differentiating equation (10) with respect to each of the unknown parameters $a_{k}, b_{k}, n_{k}, T_{w, k}$ and setting the resulting expression at zero. This yields a set of four non-linear equations. Seeking the solution is iterative and starts with an initial guess that we obtain as follows.

Firstly we ignore the dependence of the calibration coefficients on the flow temperature and determine the $\mathbf{A}_{\mathbf{k} \mathbf{0}}=\left(a_{k 0}, b_{k 0}, n_{k 0}\right)$ solution of the corresponding problem:

$$
\arg \min _{\mathbf{A}_{\mathbf{k} \mathbf{0}}} \quad\left\{\sum_{i=1}^{N_{s}}\left[M_{i, k}-f_{i, k 0}\left(U_{i}, \mathbf{A}_{\mathbf{k} \mathbf{0}}\right)\right]^{2}\right\},
$$

where $f_{i, k 0}\left(U_{i}, \mathbf{A}_{\mathbf{k} \mathbf{0}}\right)=a_{k O}+b_{k O} U_{i}^{n_{k O}}$ and $M_{i, k}=e_{i, k}^{2}$. An example of the $f_{i, k 0}$ law obtained for an overheat level $k$ is shown in figure 5 , the corresponding curve being plotted across the points corresponding to the $e_{i, k}$ values measured. The dispersion of 


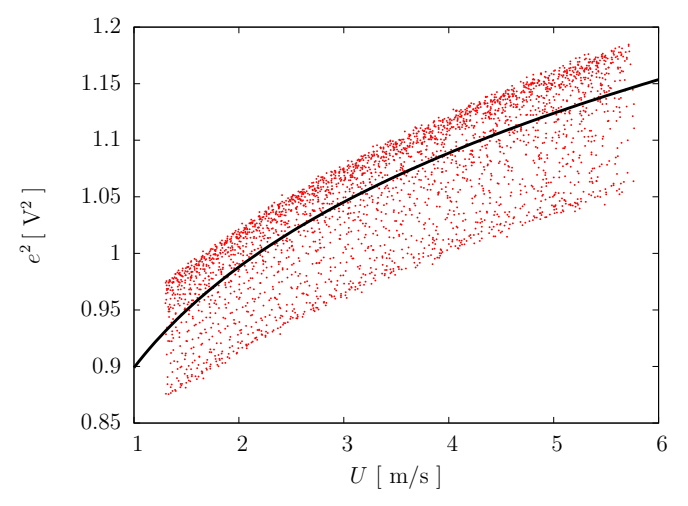

Figure 5. Variations in the squared anemometric voltage as a function of velocity and adjustment by a $f_{i, k 0}$ law (flow temperature is not considered).

the measured voltage for the same velocity value shows the sensitivity to the air temperature.

The vector $\mathbf{A}_{\mathbf{k} \mathbf{0}}=\left(a_{k 0}, b_{k 0}, n_{k 0}\right)$ is then held constant, and we determine the initial guess for the wire temperature $T_{w, k 0}$ by a linear regression according to:

$$
\frac{e_{i, k}^{2}}{f_{i, k 0}}=T_{w, k 0}-T_{i}
$$

where $\frac{e_{i, k}^{2}}{f_{i, k 0}}$ and $T_{i}$ are the variable, $U_{i}$ and $T_{i}$ being taken to be equal to the values given by the reference probes.

These provisional values of $a_{k 0}, b_{k 0}, n_{k 0}$ and $T_{w, k 0}$ are taken as the initial guess of the overall Levenberg Marquardt optimization process where all the parameters of the problem $\left(a_{k}, b_{k}, n_{k}\right.$ and $\left.T_{w, k}\right)$ are adjusted simultaneously (the variations in the flow temperature then being considered). An example of the adjustment by a law identified by this procedure for a given overheat level is shown in figure 6 where the $e_{i, k}$ values measured and the curve of the model $f_{i, k}$ are represented. The reduction in the scattering of the corrected $e_{i, k}$ values around the $f_{i, k}$ curve, as compared with figure 5 , illustrates the effectiveness of taking into account the flow temperature for the determination of a relevant calibration law.

\subsection{Computation of the instantaneous velocity-temperature pairs}

Once the calibration coefficients have been determined, we use a similar procedure to calculate the velocity and temperature $U_{i}$ and $T_{i}$ for each measurement point (corresponding to an overheat sequence) using equation (9).

For each overheat sequence $i$, we calculate the values of velocity $U_{i}$ and temperature $T_{i}$ that minimize (in the least-squares sense) an error averaged on the whole set of 


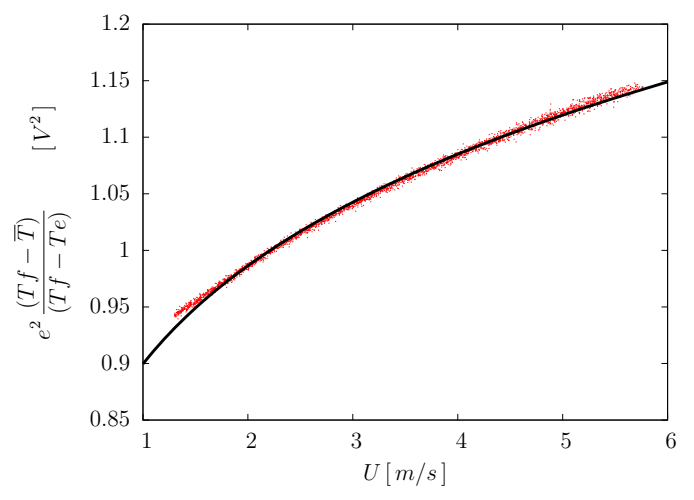

Figure 6. Variations in the corrected squared anemometric voltage as a function of velocity and adjustment by a $f_{i, k}$ law (flow temperature is considered). $\bar{T}$ denotes the average temperature during calibration.

overheat levels in use,

$$
\arg \min _{U_{i}, T_{i}} \quad\left\{\sum_{k=1}^{N_{o}}\left[M_{i, k}-f_{i, k}\left(U_{i}, T_{i}, \mathbf{A}_{\mathbf{k}}\right)\right]^{2}\right\} .
$$

This optimization problem is solved using the Levenberg-Marquardt algorithm, where $U_{i}$ and $T_{i}$ are the variables to be estimated and the calibration vector $\mathbf{A}_{\mathbf{k}}=$ $\left(a_{k}, b_{k}, n_{k}, T_{w, k}\right)$ is known.

To provide a readable representation of the optimization process involved, it should be noted that the voltage value, $e_{i, k}$, extracted from the $k^{\text {th }}$ overheat plateau inside the sequence, yields (through (13)) an infinity of $\left(U_{i}, T_{i}\right)$ solutions located on a single continuous curve $U=f(T)$. The optimization process is then equivalent to the seeking process aimed at finding, in a $(U, T)$ space, the $\left(U_{i}, T_{i}\right)$ pair that minimizes an average distance to the $k$ curves given by the whole set of $k$ overheat levels. Figure 7 gives a graphical representation of such a process.

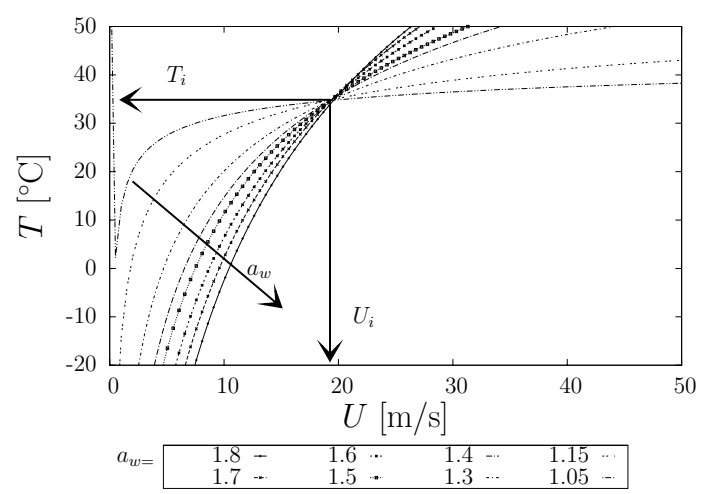

Figure 7. Illustration of $\left(U_{i}, T_{i}\right)$ solution search during the optimization procedure $\left(N_{o}=8\right)$. 
A typical example of the three dimensional surface in which the error $\epsilon_{i}=$ $\sum_{i=1}^{N_{0}}\left[M_{i, k}-f_{i, k}\left(U_{i}, T_{i}, \mathbf{A}_{\mathbf{k}}\right)\right]^{2}$ moves towards its minimum value to provide an optimized value for $U_{i}$ and $T_{i}$ is illustrated in figure 8. Such a representation illustrates our finding

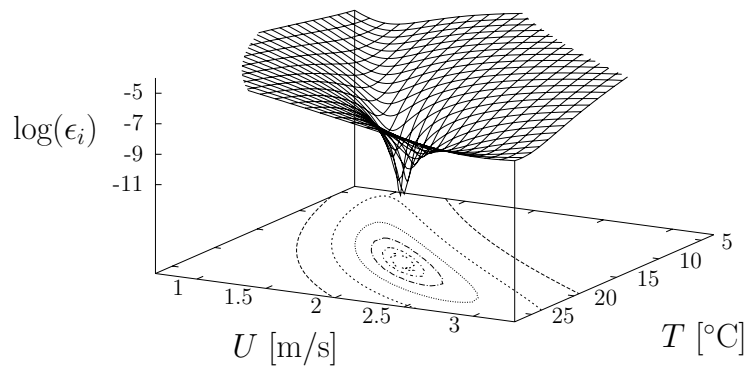

Figure 8. Three dimensional illustration of $\left(U_{i}, T_{i}\right)$ solution search.

that the risk of a local minimum in the solution zone is generally small. Provided the convergence criterion was chosen properly and the initial guess was reasonably close to the final solution, errors due to a local minimum did not occur in our experiments, achieved by:

- treating temporal sequences $e_{i, k}(t)$ sufficiently continuous in time to consider the solution at $t_{n-1}$ as a good initial guess for the solution at $t_{n}$.

- considering minimization of the error $\epsilon_{i}$ as accomplished when the relative variation in $\epsilon_{i}$ between two iterations was low enough and when values of $U_{i}$ and $T_{i}$ were stable.

\subsection{Verification of the efficiency of signal processing}

The efficiency of the signal analysis technique was checked by processing the PCTA signals acquired during the calibration experiment with this iterative approach and comparing the results (calculated velocity and temperature) to the instantaneous velocities and temperatures of the flow in the calibration test section as measured by the reference probes, namely the Pitot tube probe and the reference thermocouple. It must be noted that at this stage the uncertainty associated with the reference probes was not considered for the estimation of the uncertainty. Probability density functions (PDF) of the "errors" (deviations of the estimated values $U_{\text {est }}$ and $T_{\text {est }}$ from the reference measurements $U_{\text {ref }}$ and $T_{\text {ref }}$ ) are represented in figure 9 for a typical calibration run, with a velocity range from $0.5 \mathrm{~m} / \mathrm{s}$ to $5.5 \mathrm{~m} / \mathrm{s}$ and a temperature range from $10^{\circ} \mathrm{C}$ to $35^{\circ} \mathrm{C}$. Here the dissymetry of the PDF velocity "errors" accounts for the difference between the model (9) and the data which can be seen in figure 6 for low velocities. This bias, also observerd with the joint PDF of the errors, may lead to imperfect decoupling of the lowest velocity and temperature values in the measurement process. The standard 
a)

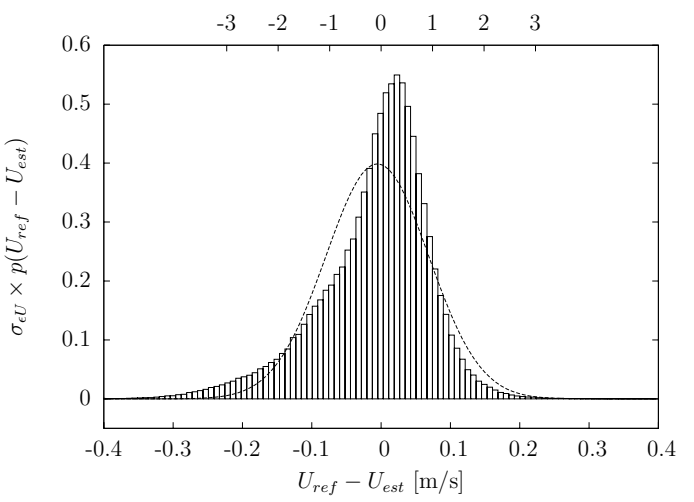

b)
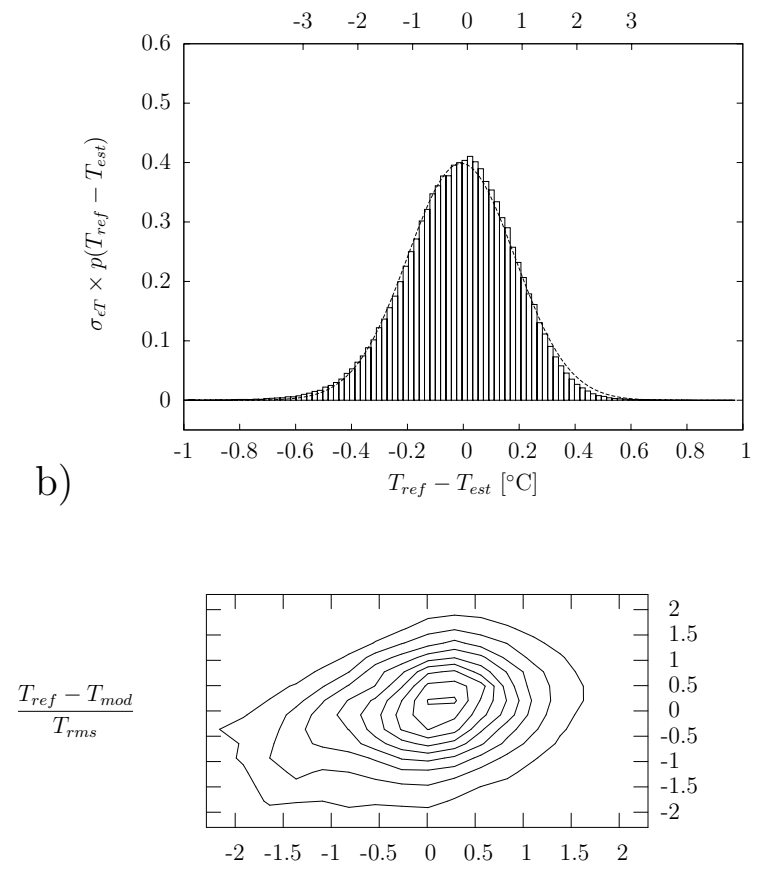

c)

$$
\frac{U_{r e f}-U_{m o d}}{U_{r m s}}
$$

Figure 9. Example of errors corresponding to difference between calibration values and corresponding values calculated with the Levenberg Marquardt algorithms: (a) velocity errors PDF, (b) temperature errors PDF, (c) Joint PDF of the errors.

deviation of the "error" is less than $0.1 \mathrm{~m} / \mathrm{s}$ for velocity and $0.2^{\circ} \mathrm{C}$ for temperature. Ideally, i.e. for a model perfectly fitting the data, as for temperature in the present study, the PDF of the "error" is symmetric and the associated standard deviation corresponds to the uncertainty of the reference probes.

The good agreement between the calculated and the reference velocities and temperatures was considered to be a good initial validation of the efficiency of our algorithms. 


\section{Measurement uncertainty}

One important stage in the design of our calibration procedure and apparatus was the evaluation and minimization of the resulting measurement uncertainty. Since the velocity and temperature measured with PCTA are determined from the output voltage through a non-linear model, we used a Monte Carlo simulation as described in [1] to evaluate the uncertainty of the PCTA velocity and temperature measurements.

Monte Carlo simulations provide an alternative approach to evaluation of uncertainty in which the propagation of uncertainties is undertaken numerically rather than analytically. This technique is able to provide much richer information by propagating the distributions rather than just the uncertainties. Confidence intervals or confidence regions can be produced from the resulting distributions.

First we estimated the uncertainty due to the calibration process. For each overheat level, we propagated the distributions of the reference velocity, the reference temperature and the PCTA voltage through the optimization solution (10) to provide distributions of the calibration vector $\mathbf{A}_{\mathbf{k}}$. The distributions of the inputs were chosen to be normal, with standard deviations determined experimentally, and equal to $0.1 \mathrm{~m} / \mathrm{s}$ for the reference velocity, $0.2 \mathrm{~K}$ for the reference temperature and $0.7 \mathrm{mV}$ for the PCTA voltage. Based on the distribution of the inputs, 1000 random fictive values were generated centered on each triplet $\left(U_{i}, T_{i}, e_{i}\right)$ recorded during the calibration stage. The procedure provided $1000 \mathbf{A}_{\mathbf{k}}$ vectors, yielding the calibration coefficients. The covariance of this output vector was fairly small, typically between $0.03 \%$ and $0.2 \%$ of its mean value. Note that this small uncertainty was mainly due to the large number of recorded triplets (around $3 \times 10^{5}$ ). For a classical calibration with 50 triplets, the error is about $8 \%$.

Next, a similar method was used to determine the uncertainty in the PCTA velocity and temperature measurements. The distributions of the calibration coefficients $\mathbf{A}_{\mathbf{k}}$ and of the PCTA voltage measurements were propagated through the optimization solution (13) to provide the distributions of velocity and temperature.

An example of the nearly Gaussian PDF built up with the values obtained by Monte Carlo simulations of the velocity and temperature at one location in a low speed mixing layer is shown in figure 10. This method was applied to calculate the uncertainty in the measurement of $\mathrm{U}$ and $\mathrm{T}$ for different calibration sample sizes from 25 to $3 \times 10^{5}$ points, with the PCTA operating in a 3 overheats configuration: as shown in figure 11, the greater the calibration sample size $N$, the more precise the $U$ and $T$ measurements. The errors evolve approximately as $1 / N$. For a sample size of $3 \times 10^{5}$ the errors in $U$ and $T$ are $1 \%$ for velocity and $0.15 \mathrm{~K}$ for temperature.

\section{Experimental results}

The PCTA method was used for the characterization of a low speed non-isothermal mixing layer. In this flow, the local shear generated by two adjacent parallel flows at different velocities was associated with a temperature difference between the 
a)
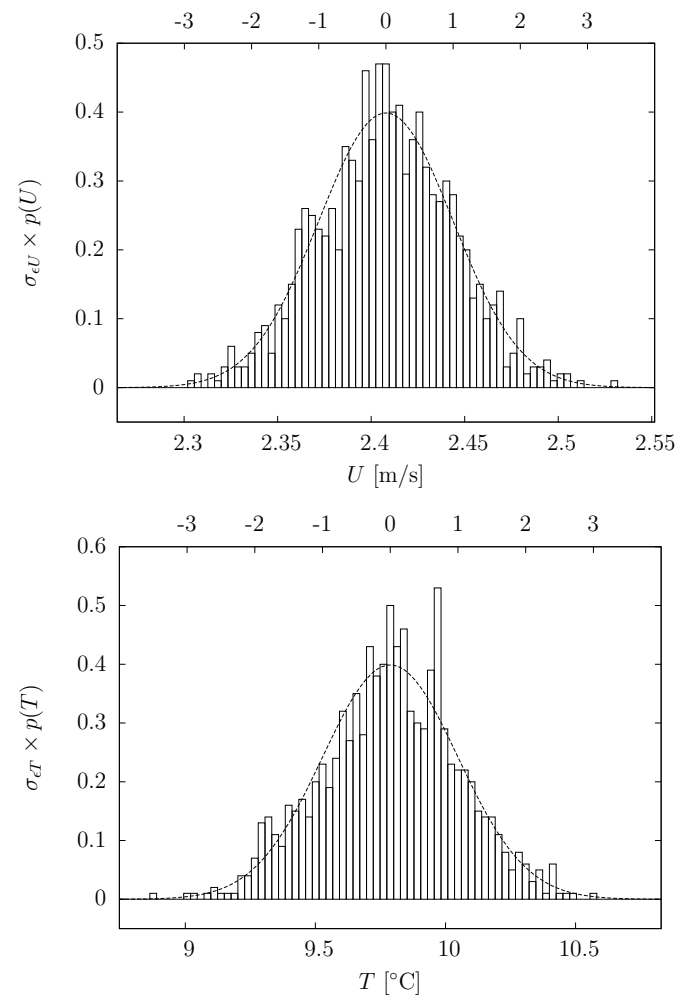

Figure 10. Example of Monte Carlo simulation results for: a) velocity, b) temperature. Each PDF is centered on the mean value for all the Monte Carlo runs (typically 1000) and the standard uncertainty is taken as equal to the standard deviation over the runs.

flows. Firstly, measurements were carried out to compare the results obtained by the PCTA method with parallel measurements obtained with two frequently used wellestablished methods, namely Constant Temperature Anemometry (CTA) and Constant Current Anemometry (CCA). Once the measurement validity had been assessed, the synchronicity of the PCTA measurements provided a conditional analysis of the interactions between velocity and temperature fluctuations in the turbulent mixing process. Figure 12 gives an example of the simultaneous outputs from the PCTA obtained at the same point in the mixing layer.

\subsection{Experimental setup}

A diagram of the flow configuration is shown in figure 13. Two parallel streams of different velocities and temperatures, generated through separate settling chambers and contraction cones, merge and initiate a mixing process behind the thin horizontal trailing edge of a $2 \mathrm{~m}$ long tapering separating plate. The $3 \mathrm{~m}$ long test section has a cross section of $1 \mathrm{~m} \times 1 \mathrm{~m}$. The origin of the coordinate system is at the center of the width of the trailing edge of the separating plate. The $x$ axis is the longitudinal streamwise direction, the $y$ axis is the upward transverse direction and the $z$ axis is the spanwise direction of the mixing layer. 


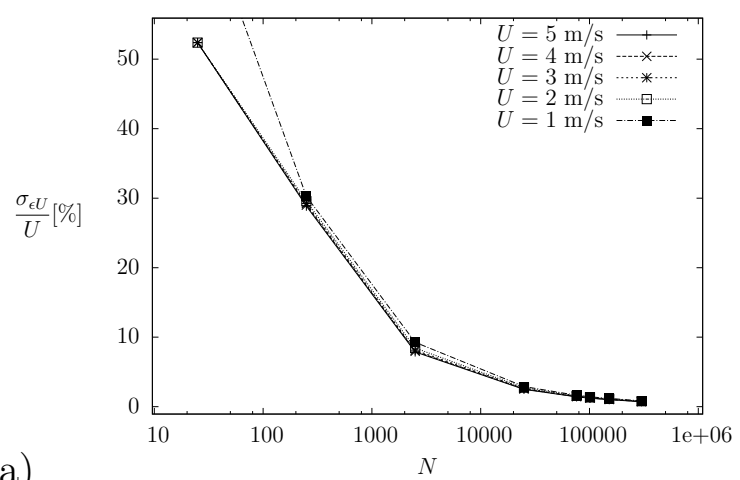

a)

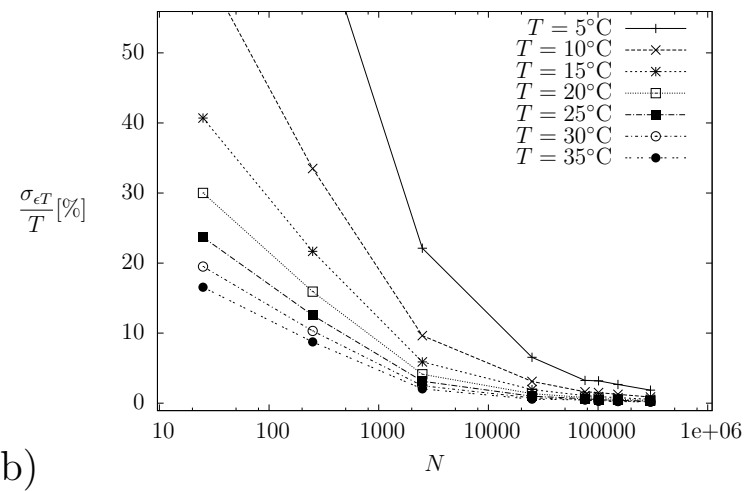

Figure 11. Example of Monte Carlo simulation results for the uncertainties of: a) velocity, b) temperature.

a)
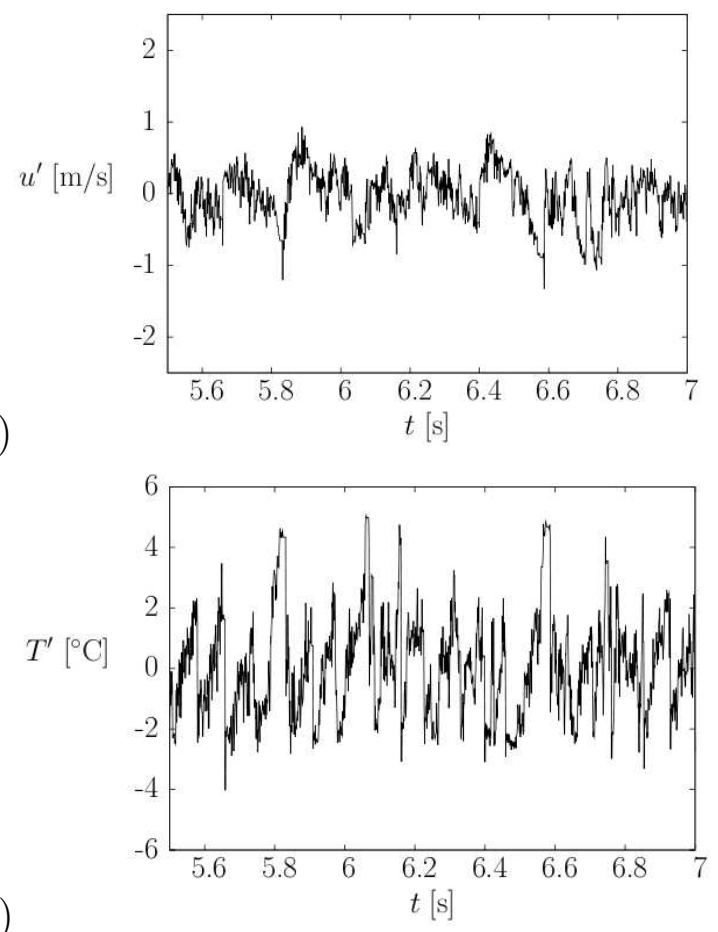

Figure 12. Simultaneous outputs from the PCTA at the same point: a) velocity fluctuations, b) temperature fluctuations. 


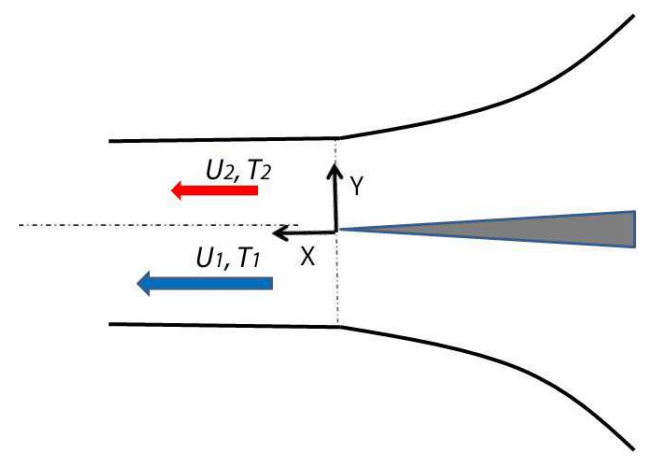

Figure 13. Diagram of flow configuration.

The bulk velocities of the lower flow $\left(U_{1}\right)$ and upper flow $\left(U_{2}\right)$ are $4 \mathrm{~m} / \mathrm{s}$ and 2 $\mathrm{m} / \mathrm{s}$, respectively. The temperatures $T_{1}$ (lower flow) and $T_{2}$ (upper flow) varied from $10^{\circ} \mathrm{C}$ to $35^{\circ} \mathrm{C}$ to study the influence of the difference in temperature $\Delta T=T_{2}-T_{1}$ on measurement accuracy.

In the experiments presented here, the PCTA system had been set with a reduced number of overheat levels. Only three overheat levels were used to separate the $U$ and $T$ information within the sequences. This reduction made possible a higher frequency response of the probe through a reduction in the sequence duration to $1 \mathrm{~ms}$. The uncertainty analysis showed that, provided the overheat ratio range was wide enough and the lowest overheat ratio small enough (typically 1.2), the accuracy of the $U$ and $T$ measurements could be maintained at a high level despite reduction in number of overheats [12]. The transverse distributions (along $y$ ) of the instantaneous velocity and temperature were measured at different streamwise locations.

\subsection{Comparison with CTA and CCA measurements}

Measurements were compared using a $2.5 \mu \mathrm{m}$ diameter hot wire probe operating in PCTA mode, and a $1.25 \mu \mathrm{m}$ diameter cold wire operating in CCA mode for temperature, and a $2.5 \mu \mathrm{m}$ diameter operation in CTA mode (with a single overheat level) for velocity. The CTA calibration involved correction of the influence of temperature obtained by applying the same calibration strategy as for the PCTA (3.1).

The mean velocity and temperature values measured by the PCTA were in good agreement with the results obtained with the CTA and the CCA, respectively, as shown in figure 14. The velocity fluctuations measured by the CTA were higher than those measured by the PCTA for the same wire diameter. Neverthless, good agreement of these values was found when normalized through division by $\left(U_{r m s}\right)_{\max }$, as shown in figure 15. A comparison of the maxima of rms values measured by PCTA and CTA, with corresponding PIV measurements, is shown in figure 16 for various streamwise positions in the self-similarity region of the mixing layer. It highlights an increase in the measurement levels of CTA velocity with the temperature difference $\Delta T$, while the PCTA measurements converge to a single value entirely independent 


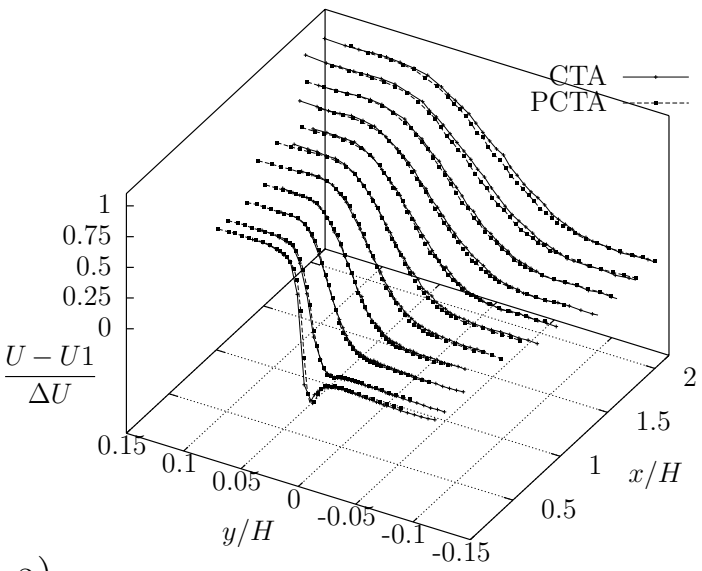

a)

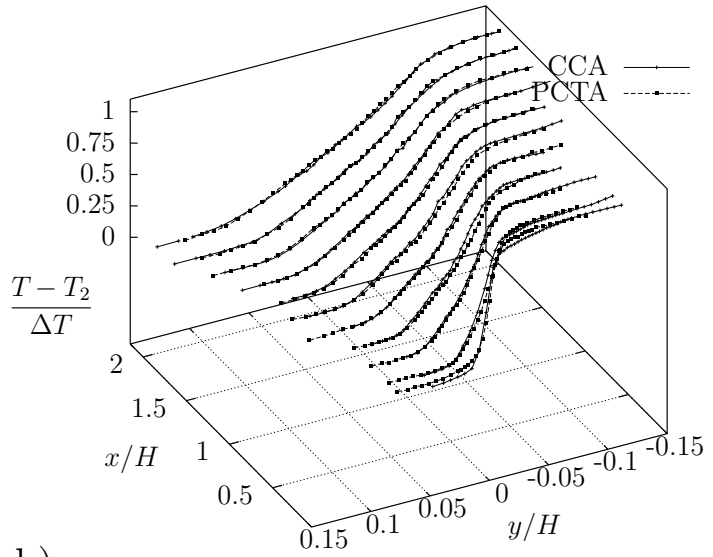

b)

Figure 14. Mean values of a) velocity and b) temperature measured by PCTA, CTA and CCA.

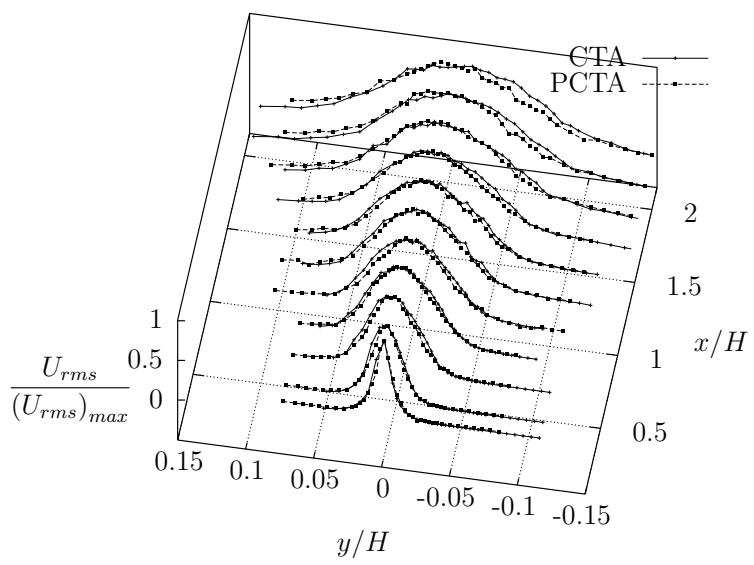

Figure 15. Normalized RMS values of velocity, comparison between PCTA and CTA. 
of $\Delta T$ and in conformity with the values given by PIV. Thus the PCTA provides

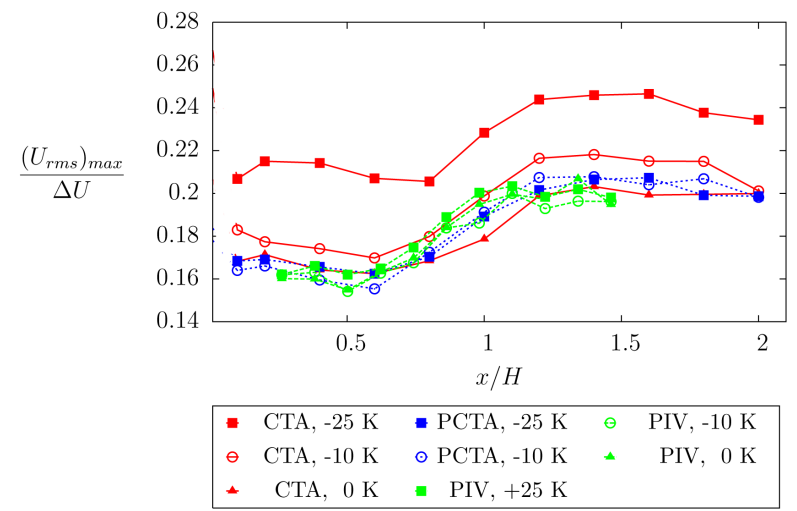

Figure 16. Normalized RMS values of velocity: PCTA, CTA and PIV measurements for different values of $\Delta T$ along the mixing layer axis in the central plane $z=0$.

good measurement of velocity fluctuations in non-isothermal situations compared to the CTA, the measurements of which are overestimated under the influence of temperature fluctuations, despite correction based on the mean instantaneous temperature values and derived from the calibration strategy described in 3.1.

When corrected for the difference between the diameters of the PCTA and CCA wires in use, measurements of the PCTA and CCA temperature fluctuations were in agreement, as shown in figure 17. This correction is based on the dependence of the probe bandwith according to wire diameter in cold wire anemometry and uses the average ratio of $\left(T_{r m s}\right)_{P C T A} /\left(T_{r m s}\right)_{C C A}$ along the centerline of the flow. Analysis of a series of transverse profiles showed that the PCTA could accurately reveal typical mixing layer profiles, with evidence of greater streamwise expansion of the thermal mixing region as compared to the dynamic region ([12]). A triple inflexion shape was found in the mean temperature profiles, in accordance with previous scalar measurements by Fiedler [7] and Masutani and Bowman [9], while the mean and rms velocity profiles depicted classical evolutions. As expected, the transverse profiles collapsed at a large downstream distance, where the flow became self-similar. The maximum value of temperature fluctuations was $T_{r m s} / \Delta T=0.25$, with a development distance around $x / \Theta_{H . V}=300$, where $\Theta_{H . V}$. was the momentum thickness of the boundary layer on the high velocity side. The peak value of the velocity fluctuations $U_{r m s} / \Delta U=0.19$ was comparable to the values obtained by Mehta [10] and Oster and Wygnanski [15], and the development distance of the flow (around $x / \Theta_{H . V .}=500$ ) fits that found in the literature for similar conditions very well $([5])$. 


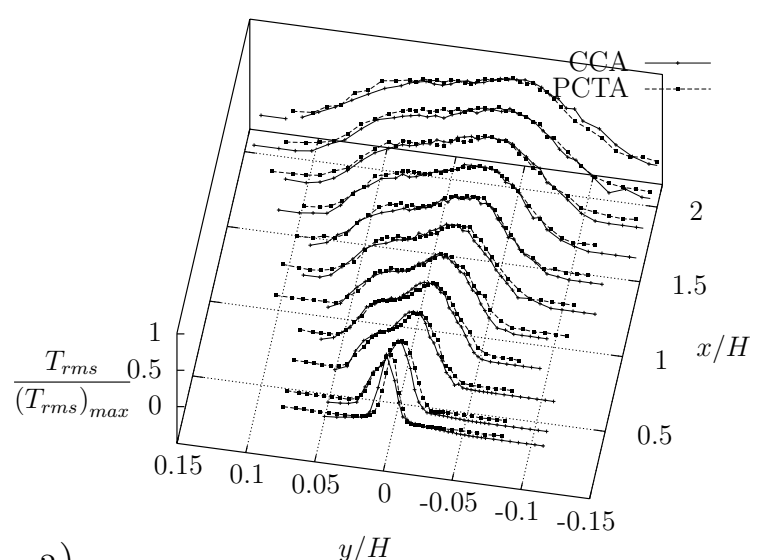

a)

$$
y / H
$$

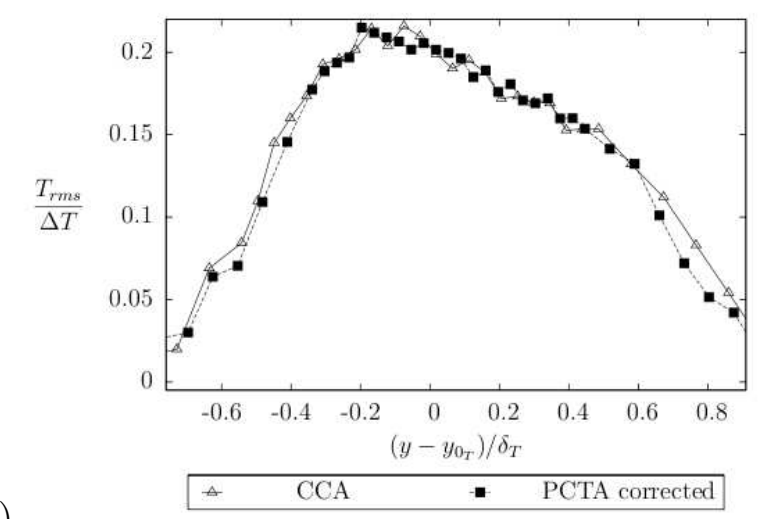

b)

Figure 17. RMS values of the temperature fluctuations measured by PCTA and CCA: a) profiles of the normalized values; b) values at a given $x$ position, with a correction applied to the PCTA data.

\subsection{Conditional analysis of PDFs}

In addition to the ability of the PCTA to identify the characteristic magnitudes of velocity and temperature, its main advantage is the synchronicity of the instantaneous velocity and temperature measurements at the same point with a relatively high frequency. From the combination of the $u^{\prime}$ and $T^{\prime}$ fluctuations obtained as separate outputs, a conditional analysis based on PDFs, temporal cross correlations and quadrant analysis could be performed to analyze the mutual interaction mechanism between these fluctuations in the mixing layer $([12,13])$. As an example of the approach applied, we give here some results of a conditional PDF analysis. Figure 18 shows the conditional PDFs of temperature obtained at different positions across the layer for a given streamwise location in the self-similarity region. This figure is composed of seven graphs from a) to g), each corresponding to a measurement point at a given transverse position $\eta_{u}$ for the streamwise position $x / \Theta_{H . V .}=593 . \quad \eta_{u}$ is a normalized value along the transverse axis $y\left(\eta_{u}=0\right.$ is the center of the dynamic mixing layer and the vorticity thickness expands from $\eta_{u}=-0.5$ to $\left.\eta_{u}=+0.5\right)$. These positions were 

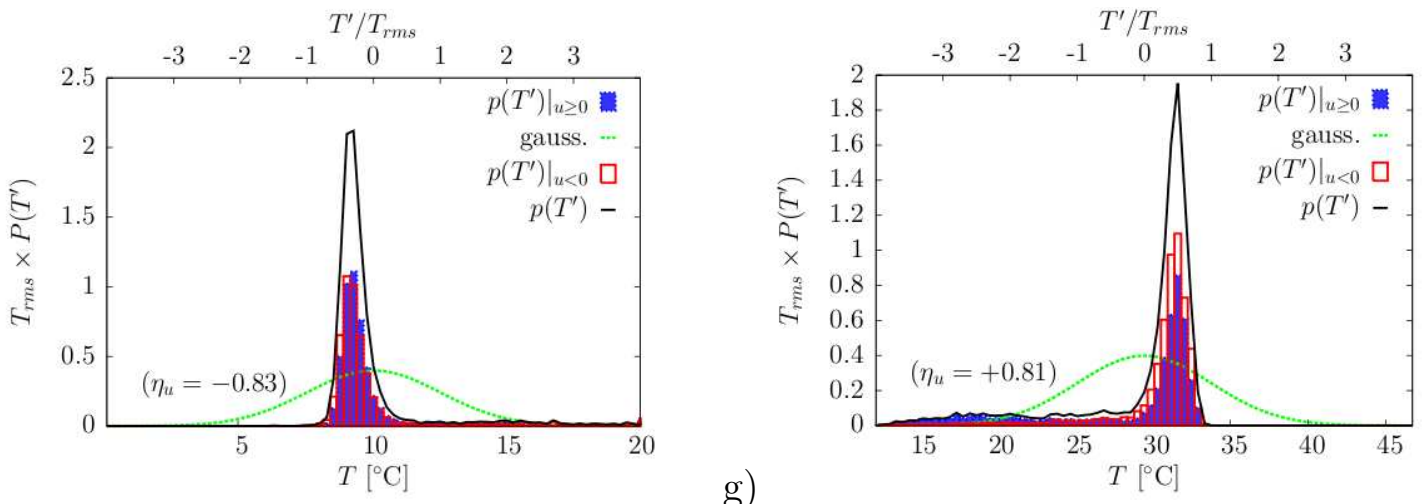

a)

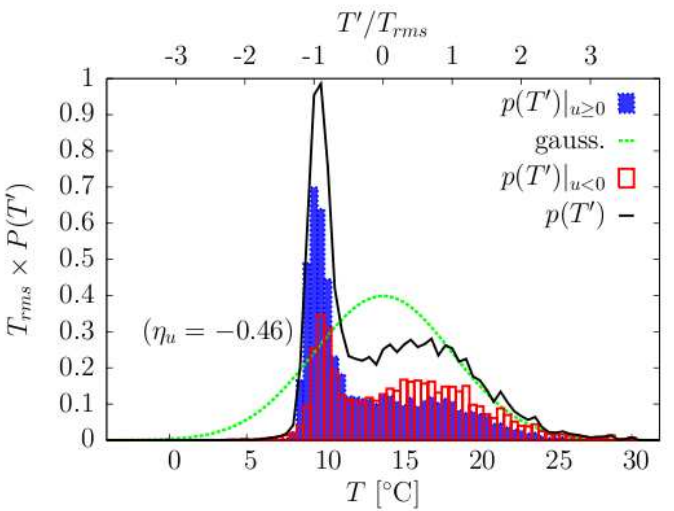

b)

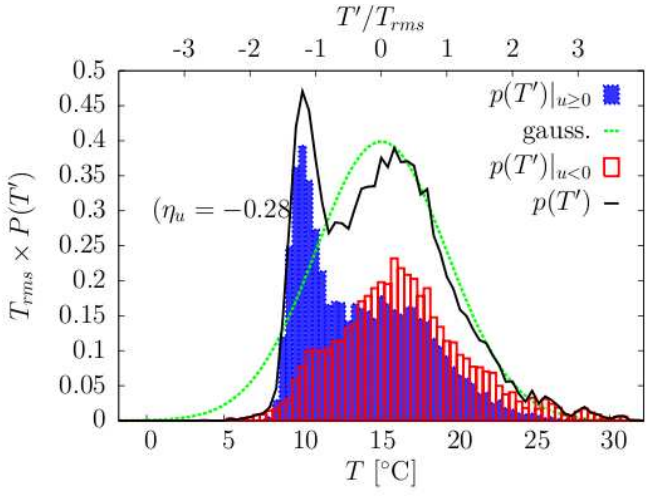

c)

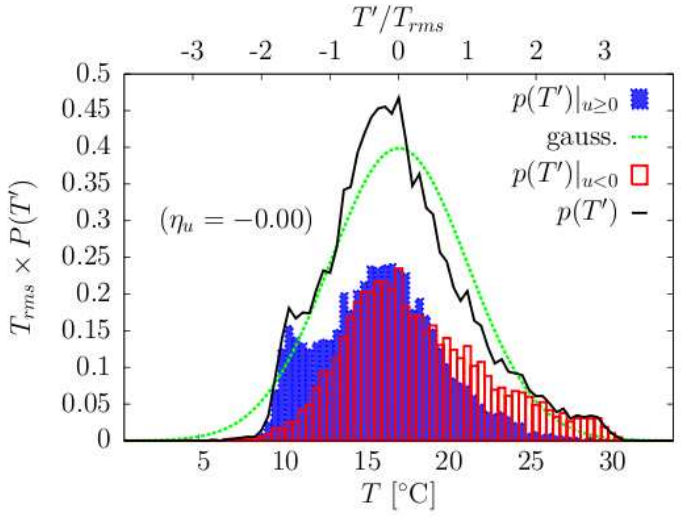

g)

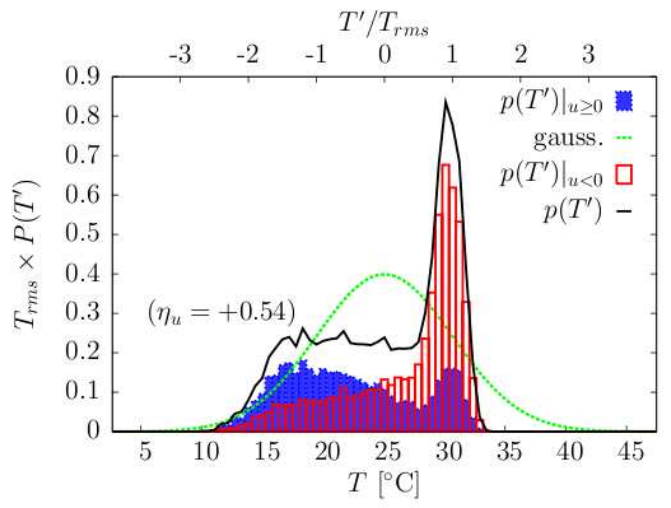

f)

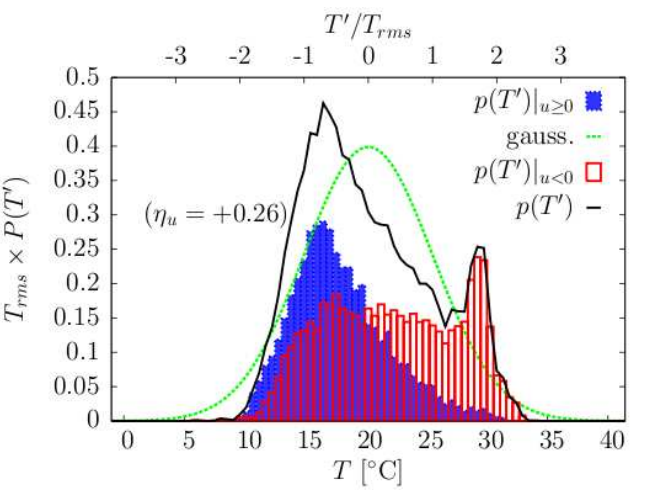

e)

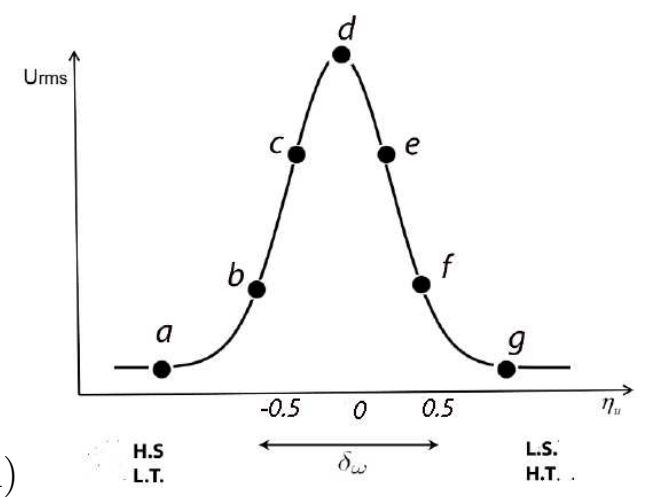

Figure 18. a-g) Variations across the mixing layer of temperature PDFs influenced by the sign of the velocity fluctuations for $\frac{U_{1}}{U_{2}}=0.50$ and $\Delta T=25 \mathrm{~K}$. The position of the corresponding measurement points is indicated in the bottom right graph. 
chosen in order to scan the breadth of the mixing layer at the given position $x$ from the cold, rapid side to the hot, slow side. In each of these graphs, four curves can be found representing the conditional PDFs $\left.p\left(T^{\prime}\right)\right|_{u^{\prime}<0}$ and $\left.p\left(T^{\prime}\right)\right|_{u^{\prime} \geq 0}$ (associated with the occurrence of negative and positive velocity fluctuations, respectively), the total PDF $p\left(T^{\prime}\right)$ and the corresponding Gaussian distribution. The tendencies generally observed, whatever the position of $x$ in the developed region, are:

- In the uniform flow region on both sides of the mixing zone (a and g), the conditional PDF curve and the overall curve show narrow peaks resulting from the weak influence of the other side of the mixing layer on this part of the flow.

- In the proximity of the edges of the mixing layer $(b$ and $f)$, PDFs are still far from gaussian curves (shown here by a dashed green line) with dissymmetry related to the non-equivalent contributions of temperature fluctuations associated with $u^{\prime} \geq 0$ or $u^{\prime}<0$. The curves show a coexistence of blobs of barely mixed fluid with more turbulent contributions (approaching gaussian levels).

- The further the curves from the edges in the direction of the mixing layer axis, the less the flow is influenced by the intermittency originating from the edges. The fluid originating from both sides of the mixing layer is in a situation of turbulent mixing, giving PDFs very close to gaussian levels.

These behaviors clarify the marching character of temperature PDFs in the self-similarity region (variation in the mode with the transverse position in the mixing layer for a given streamwise position) ([14], [2], [12]). The PCTA data made possible another type of analysis, based on quadrants, that led to description of the mechanisms associated with intermittency, with evidence of domination by saddle point ejection $([12],[13])$.

\section{Conclusion}

The development of a new anemometer allowing multiple heat sequence repetitions, and the implementation of a dynamic calibration procedure associated with a non-linear signal processing strategy, yielded new possibilities for simultaneous measurement of velocity and temperature at the same point in turbulent non-isothermal flows. The accuracy of the PCTA method was assessed with a range of velocities and temperatures corresponding to a classical turbulent thermal mixing process in low speed air flows (up to $5 \mathrm{~m} / \mathrm{s}$ and $35^{\circ} \mathrm{C}$ ). The main advantage of the method, i.e. the ability to provide the analysis of mutual interactions between velocity and temperature fluctuations, was emphasized through the example of a non-isothermal mixing layer.

\section{References}

[1] BIPM and IEC and IFCC and ILAC and ISO and IUPAC and IUPAP and OIML 2008 Supplement of ISO GUM 95, JCGM 101:2008 - Evaluation of measurement data - Supplement 1 to the Guide 
to the Expression of Uncertainty Measurement - Propagation of distributions using a Monte Carlo method

[2] Bisset, D.K 1998 Numerical simulation of heat transfer in turbulent mixing layers 13th Australasian Fluid Mechanics conference 21-24

[3] Blair, M.F. and Bennett, J.C. 1987 Hot-wire measurements of velocity and temperature fluctuations in a heated turbulent boundary layer J.Phys.E.Sci.Instrum 20 209-16

[4] Bremhorst, K. and Graham, L.J.W. 1990 A fully compensated hot/cold wire anemometer system for unsteady flow velocity and temperature measurements Meas. Sci. and Technol. 1 425-30

[5] Browand, F.K. and Latigo, B.O. 1983 Growth of two dimensional mixing layer from a turbulent and non turbulent boundary layer Physics of Fluids 22 1011-1019

[6] Bruun, H. H.1995 Hot-wire anemometry: Principles and signal analysis (Oxford University press Inc., New york, USA)

[7] Fiedler, H.E. 1974 Transport of heat across a plane turbulent mixing layer Advances in Geophysics 18 93-109

[8] Lienhard, J.H. and Helland, K.N. 1989 An experimental analysis of fluctuating temperature measurements using hot-wires at different overheats Exp. in Flu. 7 265-270

[9] Masutani, S.M. and Bowman, C.T. The structure of a chemically reacting plane mixing layer 1986 Journal of flu. Mech. 172 93-126

[10] Mehta, R.D. 1991 Effect of velocity ratio on plane mixing layer development: Influence of the splitter plate Exp. in Flu. 10 194-204

[11] Ndoye, M. and Heitz, D. and Fourment, C. and Arroyo, G. and Delville, J. 2007 Parameterizable Constant Temperature Anemometer for simultaneous velocity and temperature measurements in turbulent flows Proc. of the $8^{\text {th }}$ Int. Symp. on Experimental and Computational Aerothermodynamics of Internal Flows - Lyon

[12] Ndoye, M. 2008 Anémométrie fil chaud à température variable: application à l'étude d'une couche de mélange anisotherme Thèse de Doctorat, Université de Poitiers

[13] Ndoye, M. and Dorignac, E. and Delville, J. and Arroyo, G. 2009 Analysis of the velocity and temperature coupling in a plane mixing layer using hot wire anemometry Proceedings of the $9^{\text {th }}$ International Symposium on Turbulence Heat and Mass Transfer - Rome (ed Hanjalic, K. and Nagano, Y. and Jakirlic, K., Begell House inc.)

[14] Moser, R. D. and Rogers, M. M. 1993 The three-dimensional evolution of a plane mixing layer: pairing and transition to turbulence Journal of Flu. Mech. 247 275-320

[15] Oster, D. and Wygnanski, I. 1982 The forced mixing layer between parallel streams Journal of Flu. Mech. 123 91-130

[16] Perry, A. 1982 Hot-wire anemometry (Clarendon Press, Oxford, UK)

[17] Press, W.H. and Teutolsky, S.A. and Vetterling, W.T. and Flannery, B.P. 1992 The art of scientific computing - second edition Numerical Recipes in Fortran (Cambridge University Press)

[18] Vukoslavcevic P.V. and Wallace, J.M. 2002 The simultaneous measurement of velocity and temperature in heated turbulent air flow using thermal anemmetry Measurement Science and Technology 13 1615-24 